\title{
High-scale validity of a two-Higgs-doublet scenario: metastability included
}

\author{
Nabarun Chakrabarty ${ }^{\mathrm{a}}$, Biswarup Mukhopadhyaya ${ }^{\mathrm{b}}$ \\ Regional Centre for Accelerator-based Particle Physics, Harish-Chandra Research Institute, Chhatnag Road, Jhunsi, Allahabad 211019 , India
}

Received: 26 July 2016 / Accepted: 19 February 2017 / Published online: 10 March 2017

(C) The Author(s) 2017. This article is published with open access at Springerlink.com

\begin{abstract}
We identify regions in a Type-II two-Higgsdoublet model which correspond to a metastable electroweak vacuum with lifetime larger than the age of the universe. We analyse scenarios which retain perturbative unitarity up to grand unification and Planck scales. Each point in the parameter space is restricted using data from the Large Hadron Collider (LHC) as well as flavour and precision electroweak constraints. We find that substantial regions of the parameter space are thus identified as corresponding to metastability, which complement the allowed regions for absolute stability, for top quark mass at the high as well as low end of its currently allowed range. Thus, a two-Higgs-doublet scenario with the electroweak vacuum, either stable or metastable, can sail through all the way up to the Planck scale without facing any contradiction.
\end{abstract}

\section{Introduction}

With the observation of a scalar resonance around $125 \mathrm{GeV}$ at the LHC [1,2], and hence its identification with a Higgs boson, the particle spectrum of the standard model (SM) appears to be complete. However, issues ranging from the existence of dark matter (DM) to the pattern of the neutrino mass continue to suggest physics beyond the SM. While the quest for such new physics remains on, a rather pertinent question to ask is whether the SM by itself can ensure vacuum stability at scales above that of electroweak symmetry breaking. This is because the Higgs quartic coupling evolving via SM interactions alone tends to turn negative in between the electroweak (EW) and Planck scales, thereby making the scalar potential unbounded from below. This exact location of this instability scale crucially depends on the pole masses of the top quark and the Higgs. A recent next-to-next-to-leading order (NNLO) study [3,4] finds that absolute stability up to

\footnotetext{
a e-mail: nabarunc@hri.res.in

b e-mail: biswarup@hri.res.in
}

the Planck scale requires [3]

$$
\begin{aligned}
M_{h}[\mathrm{GeV}]> & 129.4+1.4\left(\frac{M_{t}[\mathrm{GeV}]-173.1}{0.7}\right) \\
& -0.5\left(\frac{\alpha_{s}\left(M_{Z}\right)-0.1184}{0.0007}\right) \pm 1.0_{t h} .
\end{aligned}
$$

Here, $M_{t}$ is the top quark pole mass. The updated measurements of the Higgs and top quark masses [5] hint towards a metastable vacuum scenario. Such a scenario has an additional minimum in the potential, which is deeper than the electroweak vacuum [6]. However, the lifetime of the latter is higher than the age of the universe, thus enabling the present day vacuum to be consistent with the well-tested electroweak theory.

In general, vacuum instability can be alleviated by introducing additional bosonic degrees of freedom, which can offset the downward evolution of the quartic coupling of the SM. Such a possibility has indeed been explored in the context of various non-minimal Higgs sectors. One example would be the case of the celebrated Two-Higgs-Doublet models (2HDMs). Different types of 2HDM offer interesting phenomenology at present and future colliders and, are consistent with flavour physics constraints. They are also part and parcel of supersymmetric theories. In our earlier work, we showed in the context of a Type-II 2HDM that the EW vacuum can be rendered stable till the Planck scale even for a top pole mass at the high end of the allowed band [7] Moreover, this can be achieved without running into conflict with perturbativity or unitarity at high scales, and remaining consistent with all phenomenological constraints.

A 2HDM is set apart by one more vital feature. The Yukawa couplings of the SM fermions can be different compared to the SM values, in the most general 2HDM. Thus, different values for this 'scaling factor' lead to different renormalisation group (RG) evolution trajectories inspite of keeping $M_{t}$ and other initial conditions fixed. A stable vacuum till the Planck scale is achieved with specific combinations 
of the boundary conditions, i.e., given in terms of the model parameters. The issues addressed in this paper are the following:

- Is there a possible metastable vacuum in a 2HDM? Can such a balance between the bosonic and fermionic effects be struck that indeed leads to an additional minimum of the scalar potential, while prolonging the lifetime of the EW vacuum to a safe level?

- Studies on the high-scale validity of a $2 \mathrm{HDM}$ in the past were mostly confined to investigating absolute stability [7-13]. Some studies connecting higher dimensional operators to Higgs metastability have occurred in the past $[14,15]$. Moreover, a recent study of metastable vacua was done in a rather specialised kinds of 2HDM, which is the well-known inert doublet model [16]. There, the SM-like doublet faces no alteration in its interaction strengths. It would be not only more interesting but also phenomenologically more important to identify regions with metastable vacua in a 2 HDM where Electroweak symmetry breaking (EWSB) is triggered when both doublets receive vacuum expectation values [17]. Two possibilities thus open up: (a) the scalar potential could furnish additional neutral minima around the $\mathrm{TeV}$ scale in the slice spanned by the neutral fields in the two doublets, and, (b) an additional minimum can appear when the scalar potential is improved by Renormalisation Group (RG) effects. Studies related to metastability in types (a) or (b) above have been reported with, for example, higher power terms in the potential. However, a thorough investigation of the parameter space of say, a Type-II 2HDM, is not there yet. We wish to fill this void in this work.

- As has already been mentioned, a 2HDM (of, say, Type II) allows enough of parameter space with a stable vacuum, without any new physics all the way up to the Planck scale, even if the top mass is at its upper limit. The investigation in this direction becomes complete only after checking whether and how the allowed region expands, once the possibility of a metastable electroweak vacuum is also taken into account. Here we complete the picture with such a study.

The paper has the following plan. In Sect. 2, we review the salient features of the 2HDMs. Section 3 is dedicated to a discussion of how a metastable vacuum can arise, and on the completion of its lifetime. We also present an outline of the tunnelling probability computation in the same. Section 4 presents an overall strategy on how to look for a metastable vacua, and, also an account of the various experimental and theoretical constraints taken while doing so. The numerical results are highlighted in Sect. 5 and finally the study is concluded in Sect. 6.

\section{Model features}

In the present work, we consider the most general renormalisable scalar potential for two doublets $\Phi_{1}$ and $\Phi_{2}$, each having hypercharge $(+1)$ :

$$
\begin{aligned}
V\left(\Phi_{1}, \Phi_{2}\right)= & m_{11}^{2} \Phi_{1}^{\dagger} \Phi_{1}+m_{22}^{2} \Phi_{2}^{\dagger} \Phi_{2}-m_{12}^{2}\left(\Phi_{1}^{\dagger} \Phi_{2}+\Phi_{2}^{\dagger} \Phi_{1}\right) \\
& +\frac{\lambda_{1}}{2}\left(\Phi_{1}^{\dagger} \Phi_{1}\right)^{2}+\frac{\lambda_{2}}{2}\left(\Phi_{2}^{\dagger} \Phi_{2}\right)^{2}+\lambda_{3} \Phi_{1}^{\dagger} \Phi_{1} \Phi_{2}^{\dagger} \Phi_{2} \\
& +\lambda_{4} \Phi_{1}^{\dagger} \Phi_{2} \Phi_{2}^{\dagger} \Phi_{1}+\frac{\lambda_{5}}{2}\left[\left(\Phi_{1}^{\dagger} \Phi_{2}\right)^{2}+\left(\Phi_{2}^{\dagger} \Phi_{1}\right)^{2}\right] \\
& +\lambda_{6} \Phi_{1}^{\dagger} \Phi_{1}\left(\Phi_{1}^{\dagger} \Phi_{2}+\Phi_{2}^{\dagger} \Phi_{1}\right) \\
& +\lambda_{7} \Phi_{2}^{\dagger} \Phi_{2}\left(\Phi_{1}^{\dagger} \Phi_{2}+\Phi_{2}^{\dagger} \Phi_{1}\right) .
\end{aligned}
$$

This scenario in general has the possibility of CP-violation in the scalar sector [18,19], through the phases in $m_{12}, \lambda_{5}, \lambda_{6}$ and $\lambda_{7}$. We choose $m_{12}$ to be real here; moreover, the terms proportional to $\lambda_{6}$ and $\lambda_{7}$ have been neglected in the present study.

For the Yukawa part, we adopt the well-known 'Type-II 2HDM' in which the bottom-type quarks and the charged leptons couple to $\phi_{1}$ and the up-type quarks couple to $\phi_{2}$. This particular scheme can be implemented by introducing a $\mathbb{Z}_{2}$ symmetry that demands $\Phi_{1} \rightarrow-\Phi_{1}$ and $\Phi_{2} \rightarrow \Phi_{2}$. While the primary motivation of the above is to suppress flavour changing neutral currents (FCNC) [20-22], it reduces the number of free parameters in the Yukawa sector ${ }^{1}$ As a consequence, it becomes easier to throw light on the scalar sector in an analysis using the renormalisation group equations (RGEs).

Minimisation of the scalar potential in Eq. (2.1) leads to

$$
\left\langle\Phi_{1}\right\rangle=\left(\begin{array}{c}
0 \\
\frac{v_{1}}{\sqrt{2}}
\end{array}\right), \quad\left\langle\Phi_{2}\right\rangle=\left(\begin{array}{c}
0 \\
v_{2} \\
\frac{\sqrt{2}}{\sqrt{2}}
\end{array}\right),
$$

where the vacuum expectation values are often expressed in terms of the $M_{Z}$ and the ratio

$\tan \beta=\frac{v_{2}}{v_{1}}$.

We parametrise the doublets as

$\Phi_{i}=\frac{1}{\sqrt{2}}\left(\begin{array}{c}\sqrt{2} w_{i}^{+} \\ v_{i}+h_{i}+i z_{i}\end{array}\right)$ for $i=1,2$

Since the basis used in $V\left(\Phi_{1}, \Phi_{2}\right)$ allows for mixing between the two doublets, the physical states are obtained by diagonalising the charged and neutral scalar mass matrices. There

\footnotetext{
${ }^{1}$ It was reported in $[23,24]$ that the FCNCs are absent if a given fermion generation couples to any one of the doublets. In this connection, it was revealed rather recently [25] that the FCNCs are stable under the renormalisation group.
} 
are then altogether eight mass eigenstates, three of which become the longitudinal components of the $W^{ \pm}$and $Z$ gauge bosons. Of the remaining five, there is a mutually conjugate pair of charged scalars $\left(H^{ \pm}\right)$, two neutral CP-even scalars $(H, h)$ and a neutral pseudoscalar $(A)$, given that there is no CP-violation. Otherwise, a further mixing between $(H, h)$ and $A$ becomes unavoidable. The compositions of the mass eigenstates $H$ and $h$ indeed depend on the mixing angle $\alpha$.

The quartic couplings are conveniently expressed in terms of the physical masses and mixing angles, thus:

$$
\begin{aligned}
& \lambda_{1}=\frac{1}{v^{2} c_{\beta}^{2}}\left(c_{\alpha}^{2} m_{H}^{2}+v^{2} s_{\alpha}^{2} m_{h}^{2}-m_{12}^{2} \frac{s_{\beta}}{c_{\beta}}\right), \\
& \lambda_{2}=\frac{1}{v^{2} s_{\beta}^{2}}\left(s_{\alpha}^{2} m_{H}^{2}+v^{2} c_{\alpha}^{2} m_{h}^{2}-m_{12}^{2} \frac{c_{\beta}}{s_{\beta}}\right), \\
& \lambda_{3}=\frac{2 m_{H^{+}}^{2}}{v^{2}}+\frac{s_{2 \alpha}}{v^{2} s_{2 \beta}}\left(m_{H}^{2}-m_{h}^{2}\right)-\frac{m_{12}^{2}}{v^{2} s_{\beta} c_{\beta}}, \\
& \lambda_{4}=\frac{1}{v^{2}}\left(m_{A}^{2}-2 m_{H^{+}}^{2}\right)+\frac{m_{12}^{2}}{v^{2} s_{\beta} c_{\beta}}, \\
& \lambda_{5}=\frac{m_{12}^{2}}{v^{2} s_{\beta} c_{\beta}}-\frac{m_{A}^{2}}{v^{2}} .
\end{aligned}
$$

\section{The computation of tunnelling probability}

The existence of a large number of scalar degrees of freedom makes the vacuum landscape of a 2 HDM more elaborate and intriguing compared to the SM. Here we are confining ourselves to the situation when the vacuum breaks neither electric charge nor CP. Under such circumstances, the EWSB conditions appear as

$$
\begin{aligned}
& m_{11}^{2} v_{1}=m_{12}^{2} v_{2}-\frac{1}{2} \lambda_{1} v_{1}^{3}-\frac{1}{2}\left(\lambda_{3}-\lambda_{4}+\lambda_{5}\right) v_{1} v_{2}^{2} \\
& m_{22}^{2} v_{2}=m_{12}^{2} v_{1}-\frac{1}{2} \lambda_{2} v_{2}^{3}-\frac{1}{2}\left(\lambda_{3}-\lambda_{4}+\lambda_{5}\right) v_{2} v_{1}^{2}
\end{aligned}
$$

It has been reported in [26] that the above conditions can lead to several solutions, and at most two non-degenerate minima. In other words, apart from the EW minimum in which the universe currently resides $\left(v_{1}^{2}+v_{2}^{2}=246 \mathrm{GeV}^{2}\right.$, named $N$ ), there exists another minimum somewhere around $\left(v_{1}^{2}+v_{2}^{2} \neq 246 \mathrm{GeV}^{2}\right.$, named $\left.N^{\prime}\right)$. Reference [26] finds the difference of depths of the tree-level scalar potential at the two minima to be,

$V_{N^{\prime}}-V_{N}=\frac{m_{12}^{2}}{4 v_{1} v_{2}}\left(1-\frac{v_{1} v_{2}}{v_{1}^{\prime} v_{2}^{\prime}}\right)^{2}\left(v_{1} v_{2}^{\prime}-v_{2} v_{1}^{\prime}\right)^{2}$

Thus there exists the tantalizing possibility that the 2HDM offers such parameter points for which a neighbouring vacuum could actually be deeper than the one which corresponds to the observed $W-$ and $Z-$ boson masses. The EW minimum then loses its status as the global minimum and has been termed the panic vacuum in [26]. In those cases, computing the lifetime of tunnelling to the non-EW minimum from the EW one becomes the pertinent task. If the tunnelling lifetime turn out to be higher than the age of the universe, the non-EW minimum cannot be ruled out. However, thanks to the data from the LHC on Higgs signal strengths, the model points admitting $V_{N^{\prime}}-V_{N}<0$ are more or less ruled out $[26,27]$. However, a new landscape of vacua can still open up if one investigates the RG-improved effective potential in place of the bare tree-level one. In the context of the SM, it can be understood as follows: The SM quartic coupling turns negative at some energy scale $10^{8-11} \mathrm{GeV}$ (exact location of the scale depends on the choice of the initial conditions), after which it again starts rising owing to the bosonic effects counterbalancing the negative top-Yukawa drag. The consequence of this is the emergence of a new minimum beyond the scale where the quartic coupling first becomes negative. It should be noted that the direction of the EW vacuum uniquely decides the direction in which the high-scale vacuum is formed.

In a $2 \mathrm{HDM}$, on the other hand, one has to handle the additional complication of having a higher number of field directions. In addition, the effects of the various interaction terms make it imperative to incorporate the effects of radiative corrections induced by the 2HDM. Therefore, we choose to analyse the one-loop corrected effective potential [28] in place of the tree-level potential. One thus writes

$V_{\text {eff }}\left(h_{1}, h_{2}\right)=V_{\text {tree }}\left(h_{1}, h_{2}\right)+V_{\text {lloop }}\left(h_{1}, h_{2}\right)$.

Here, $V_{\text {tree }}\left(h_{1}, h_{2}\right)$ and $V_{1 \text { loop }}\left(h_{1}, h_{2}\right)$ denote the tree-level and one-loop parts of the effective potential, calculated along the $h_{1}-h_{2}$ subspace.

For example, the tree-level potential reads

$$
\begin{aligned}
V_{\text {tree }}\left(h_{1}, h_{2}\right)= & \frac{1}{2} m_{11}^{2} h_{1}^{2}+\frac{1}{2} m_{22}^{2} h_{2}^{2}+m_{12}^{2} h_{1} h_{2} \\
& +\frac{\lambda_{1}}{8} h_{1}^{4}+\frac{\lambda_{2}}{8} h_{2}^{4}+\frac{\lambda_{3}+\lambda_{4}+\lambda_{5}}{4} h_{1}^{2} h_{2}^{2} .
\end{aligned}
$$

In the $h_{1}-h_{2}$ plane, it has the following expression [29]:

$$
\begin{aligned}
V_{1 \text { loop }}\left(h_{1}, h_{2}\right)= & \frac{1}{64 \pi^{2}} \sum_{i} n_{i} M_{i}^{4}\left(h_{1}, h_{2}\right) \\
& \times\left[\ln \left(\frac{M_{i}^{4}\left(h_{1}, h_{2}\right)}{\mu^{2}}\right)-c_{i}\right] .
\end{aligned}
$$

Here $n_{i}$ refers to the number of degrees of freedom for the $i$ th field and the $c_{i}$ are constants whose values depend on the regularisation scheme adopted. To list the constants explicitly, $n_{W}=6, n_{Z}=3, n_{t}=-12, n_{h}=1$; and $c_{W}=\frac{5}{6}, c_{Z}=\frac{5}{6}$, $c_{t}=\frac{3}{2}, c_{h}=\frac{3}{2}$. Moreover, $\mu$ refers to the renormalisation 
scale emerging as an artefact of dimensional regularisation. The $M_{i}^{2}\left(h_{1}, h_{2}\right)$ represent the scale dependent mass squared.

The main theme of this work is to investigate possible high-scale vacua in the context of 2HDM using the general prescription suggested by Coleman. $V_{\text {eff }}\left(h_{1}, h_{2}\right)$ depends on two variables, and hence, determining a classical solution interpolating the two vacua, even numerically, becomes an extremely challenging task. Furthermore, a generic classical path may not qualify as a "bounce" [30,31], i.e., it might not pass through the top of a barrier separating two vacua. Coleman's prescription does not apply in such a case. However, one can always choose to look for additional minima along a particular ray in the $h_{1}-h_{2}$ plane. In this approximation, the effective potential is reduced to a function of a single variable again (the particular linear combination of $h_{1}$ and $h_{2}$ ). In models such as Type-I or Type-II $2 \mathrm{HDM}$, the $Z_{2}$ symmetry of the Yukawa interactions implies that the top quark always couples to $\Phi_{2}$. Thus it is the coupling $\lambda_{2}$ that experiences the maximum downward pull due to the Yukawa interactions and can consequently can turn negative at high scales in spite of starting with a positive value at the input scale. It therefore makes sense to look for additional minima in the $h_{2}$ direction only. This approach is similar to what [16] opts in the context of an inert doublet model.

We study the behaviour of the $V_{\text {eff }}\left(h_{1}, h_{2}\right)$ in the limit where $h_{1} \simeq v$ and $h_{2}>>h_{1}, m_{12}$. In this limit, the squared masses have the following simplified expressions:

$$
\begin{aligned}
m_{H_{1}}^{2}\left(h_{2}\right) & \simeq \frac{1}{2}\left(\lambda_{3}+\lambda_{4}+\lambda_{5}\right) h_{2}^{2}, \\
m_{H_{2}}^{2}\left(h_{2}\right) & \simeq \frac{3}{2} \lambda_{2} h_{2}^{2}, \\
m_{A_{1}}^{2}\left(h_{2}\right) & \simeq \frac{1}{2}\left(\lambda_{3}+\lambda_{4}-\lambda_{5}\right) h_{2}^{2}, \\
m_{A_{2}}^{2}\left(h_{2}\right) & \simeq \frac{1}{2} \lambda_{2} h_{2}^{2}, \\
m_{H_{1}^{+}}^{2}\left(h_{2}\right) & \simeq \frac{1}{2} \lambda_{3} h_{2}^{2}, \\
m_{H_{2}^{+}}^{2}\left(h_{2}\right) & \simeq \frac{1}{2} \lambda_{2} h_{2}^{2}, \\
m_{t}^{2}\left(h_{2}\right) & \simeq \frac{1}{2} y_{t}^{2} h_{2}^{2}, \\
m_{W}^{2}\left(h_{2}\right) & \simeq \frac{1}{4} g^{2} h_{2}^{2}, \\
m_{Z}^{2}\left(h_{2}\right) & \simeq \frac{1}{4}\left(g^{2}+g^{\prime 2}\right) h_{2}^{2} .
\end{aligned}
$$

All running couplings are evaluated at the scale $\mu \simeq h_{2}$. Under all these approximations, the real part of the one-loop corrected potential takes the form

$$
V_{\text {eff }}\left(h_{2}\right) \simeq \frac{\lambda_{2}^{\text {eff }}}{8} h_{2}^{4}
$$

where,

$$
\begin{aligned}
\lambda_{2}^{\mathrm{eff}}\left(h_{2}\right) \simeq & \lambda_{2}\left(h_{2}\right)+\frac{1}{64 \pi^{2}}\left[2\left(\lambda_{3}+\lambda_{4}+\lambda_{5}\right)^{2}\right. \\
& \times\left(\ln \frac{\lambda_{3}+\lambda_{4}+\lambda_{5}}{2}-\frac{3}{2}\right)+18 \lambda_{2}^{2}\left(\ln \frac{3 \lambda_{2}}{2}-\frac{3}{2}\right) \\
& +2\left(\lambda_{3}+\lambda_{4}-\lambda_{5}\right)^{2}\left(\ln \frac{\lambda_{3}+\lambda_{4}-\lambda_{5}}{2}-\frac{3}{2}\right) \\
& +2 \lambda_{3}^{2}\left(\ln \frac{\lambda_{3}}{2}-\frac{3}{2}\right)+6 \lambda_{2}^{2}\left(\ln \frac{\lambda_{3}}{2}-\frac{3}{2}\right) \\
& +3 g^{4}\left(\ln \frac{g^{2}}{4}-\frac{5}{6}\right)+\frac{3}{2}\left(g^{2}+g^{\prime 2}\right)^{2} \\
& \left.\times\left(\ln \frac{g^{2}+g^{\prime 2}}{4}-\frac{5}{6}\right)-24 y_{t}^{4}\left(\ln \frac{y_{t}^{2}}{2}-\frac{3}{2}\right)\right]
\end{aligned}
$$

where the term in square brackets refers to the finite correction generated by the Coleman-Weinberg mechanism. We find that highly sub-dominant in our calculations.

In this limit, the probability of tunnelling to the deeper vacuum is given by [6]

$p=T_{U}^{4} \mu^{4} e^{-\frac{8 \pi^{2}}{3 \mid \lambda_{2}^{\text {eff }}}}$.

Here one fixes $\mu$ to the scale where the probability is maximised, and, it turns out that $\frac{\mathrm{d} \lambda_{2}}{\mathrm{~d} \log (\mathrm{Q})}=0$ at $Q=\mu$. Using $T_{U} \simeq 10^{10} \mathrm{yr}$ and requiring that the vacuum tunnelling lifetime is always higher than the lifetime of the universe amounts to the following condition [6]:

$\lambda_{2}^{\mathrm{eff}}\left(h_{2}\right) \geq \frac{-0.065}{1-0.01 \ln (v / \mu)}$.

It may be noted that we have accepted $\lambda_{2}$ turning negative in the $h_{2}$ direction as the sole condition for the loss of stability of the EW vacuum. There is in general an extended set of conditions for stability in a 2HDM [32]. However, one can easily verify that the remaining conditions for stability in a $2 \mathrm{HDM}$ are violated, if at all, at low scale itself. Such a violation, on the other hand, leads to the disappearance of the EW minimum as a whole. This cannot be a situation appropriate for metastability, and therefore the conditions other than $\lambda_{2}<0$ need not be used as signs for loss of stability.

\section{A metastable vacuum and the 2HDM parameter space}

\subsection{Analysis strategy}

As has already been emphasised in the previous section, a look-out for an additional vacuum at high scales requires one to study the evolution of the various interaction strengths 
under RG equations. The values of the quartic couplings at the electroweak scale are, of course, connected with the masses and mixing angles in the scalar sector. A careful measurement of the signal strengths of the $125 \mathrm{GeV}$ at the LHC has revealed that the resonance has couplings strikingly similar to the SM ones. These observations have their ramifications on the 2HDM parameter space. Thus, together with the requirement of having $m_{h} \simeq 125 \mathrm{GeV}$, we also arrange for $\beta-\alpha \simeq \frac{\pi}{2}$, in order to comply with these results from the LHC $\left(\beta-\alpha=\frac{\pi}{2}\right.$ is the well-known alignment limit $[33,34]$ in a 2HDM, in which the couplings of $h$ to fermions and gauge bosons become exactly equal to the SM ones). We choose to describe a 2HDM model point in terms of the parameters $\left(\tan \beta, m_{h}, m_{H}, m_{A}, m_{H^{+}}, \alpha\right)$. There are additional constraints to satisfy as outlined in the next few subsections.

\subsubsection{Perturbativity, unitarity and vacuum stability}

For the $2 \mathrm{HDM}$ to remain a perturbative quantum field theory at a given energy scale, one must impose the conditions $\left|\lambda_{i}\right| \leq 4 \pi(i=1, \ldots, 7)$ and $\left|y_{i}\right| \leq \sqrt{4 \pi}(i=t, b, \tau)$ at that scale. ${ }^{2}$ This translates into upper bounds on the running couplings at low as well as high scales.

A more sophisticated version of such bounds comes from the requirement of partial wave unitarity in longitudinal gauge boson scattering. The $2 \rightarrow 2$ amplitude matrix corresponding to scattering of the longitudinal components of the gauge bosons can be mapped to a corresponding matrix for the scattering of the Goldstone bosons [35-38]. The theory respects unitarity if each eigenvalue of the aforementioned amplitude matrix does not exceed $8 \pi$.

$$
\begin{aligned}
a_{ \pm} & =\frac{3}{2}\left(\lambda_{1}+\lambda_{2}\right) \pm \sqrt{\frac{9}{4}\left(\lambda_{1}-\lambda_{2}\right)^{2}+\left(2 \lambda_{3}+\lambda_{4}\right)^{2}}, \\
b_{ \pm} & =\frac{1}{2}\left(\lambda_{1}+\lambda_{2}\right) \pm \sqrt{\frac{1}{4}\left(\lambda_{1}-\lambda_{2}\right)^{2}+\lambda_{4}^{2}} \\
c_{ \pm} & =d_{ \pm}=\frac{1}{2}\left(\lambda_{1}+\lambda_{2}\right) \pm \sqrt{\frac{1}{4}\left(\lambda_{1}-\lambda_{2}\right)^{2}+\lambda_{5}^{2}} \\
e_{1} & =\left(\lambda_{3}+2 \lambda_{4}-3 \lambda_{5}\right) \\
e_{2} & =\left(\lambda_{3}-\lambda_{5}\right) \\
f_{1} & =f_{2}=\left(\lambda_{3}+\lambda_{4}\right) \\
f_{+} & =\left(\lambda_{3}+2 \lambda_{4}+3 \lambda_{5}\right) \\
f_{-} & =\left(\lambda_{3}+\lambda_{5}\right) .
\end{aligned}
$$

When the quartic part of the scalar potential preserves $\mathrm{CP}$ and $\mathbb{Z}_{2}$ symmetries, the aforementioned eigenvalues are discussed in $[35,36,39]$.

\footnotetext{
2 The conditions are slightly different for the two types of couplings. The reason becomes clear if we note that the perturbative expansion parameter for $2 \rightarrow 2$ processes driven by the quartic couplings is $\lambda_{i}$. The corresponding parameter for Yukawa-driven scattering processes is $\left|y_{i}\right|^{2}$.
}

The condition to be discussed next is that of vacuum stability. For the scalar potential of a theory to be stable, it must be bounded from below in all possible directions. Avoiding such a possibility of a negative quartic coupling till any given scale ensures stability of the vacuum up to that scale. Demanding high-scale positivity of the $2 \mathrm{HDM}$ potential along various directions in the field space leads to the following conditions on the scalar potential [32,40-42]:

$\operatorname{vsc} 1: \quad \lambda_{1}>0$,

$\operatorname{vsc} 2: \quad \lambda_{2}>0$,

$\operatorname{vsc} 3: \lambda_{3}+\sqrt{\lambda_{1} \lambda_{2}}>0$,

$\operatorname{vsc} 4: \quad \lambda_{3}+\lambda_{4}-\left|\lambda_{5}\right|+\sqrt{\lambda_{1} \lambda_{2}}>0$.

When a second vacuum arises, we go for a test of metastability of the electroweak vacuum using vsc 2 alone. The reason for this has already been discussed at the end of Sect. 3.

\subsubsection{Oblique parameters and flavour constraints}

A 2HDM contributes to the electroweak precision observables through the participation of the additional scalars in loops. For example, the oblique $S, T$ and $U$ parameters [43] receive contributions $\Delta S, \Delta T$ and $\Delta U$, respectively, from the 2HDM [44]. The most constraining amongst these is $\Delta T$, which reads $[45,46]$

$$
\begin{aligned}
\Delta T= & F\left(m_{H^{+}}^{2}, m_{H}^{2}\right)+F\left(m_{H^{+}}^{2}, m_{A}^{2}\right)+c_{\beta-\alpha}^{2} F\left(m_{H^{+}}^{2}, m_{h}^{2}\right) \\
& +s_{\beta-\alpha}^{2} F\left(m_{H^{+}}^{2}, m_{H}^{2}\right)-F\left(m_{H}^{2}, m_{A}^{2}\right) \\
& -c_{\beta-\alpha}^{2} F\left(m_{h}^{2}, m_{A}^{2}\right)-s_{\beta-\alpha}^{2} F\left(m_{H}^{2}, m_{A}^{2}\right) \\
& +3 c_{\beta-\alpha}^{2}\left(F\left(m_{Z}^{2}, m_{H}^{2}\right)-F\left(m_{W}^{2}, m_{H}^{2}\right)\right) \\
& -3 c_{\beta-\alpha}^{2}\left(F\left(m_{Z}^{2}, m_{h}^{2}\right)-F\left(m_{W}^{2}, m_{h}^{2}\right)\right) .
\end{aligned}
$$

Here

$$
\begin{aligned}
& F\left(m_{1}^{2}, m_{2}^{2}\right) \\
& \equiv \begin{cases}\frac{m_{1}^{2}+m_{2}^{2}}{2}-\frac{m_{1}^{2} m_{2}^{2}}{m_{1}^{2}-m_{2}^{2}} \ln \frac{m_{1}^{2}}{m_{2}^{2}} ; & m_{1}^{2} \neq m_{2}^{2}, \\
0 & m_{1}^{2}=m_{2}^{2} .\end{cases}
\end{aligned}
$$

An essential input in our analysis is the existing experimental constraint on the $T$ parameter, namely, $\Delta T=$ $0.05 \pm 0.12$ following [47], $\Delta T$ being the departure from the SM contribution. Therefore, we have filtered all points in our parameter space through this constraint and retained only those points that survive it. On the restrictions coming from the flavour physics side, measurement of the $b \rightarrow s \gamma$ leads to $m_{H^{+}} \geq 315 \mathrm{GeV}$ in the case of the Type-II 2HDM [48]. In the case of Type I, there is no such lower bound. The constraint $m_{H^{+}} \geq 80 \mathrm{GeV}$ originating from direct searches, however, still persists. 
Table 1 Benchmark points chosen to illustrate the behaviour under RG. $\Lambda$ denotes the maximum extrapolation scale up to which perturbativity remains intact. The

stability/metastability of the EW vacuum corresponding to these benchmarks is dictated by the value of $M_{t}$ taken

\begin{tabular}{lcccccl}
\hline Benchmark & Tan $\beta$ & $m_{H}(\mathrm{GeV})$ & $m_{A}(\mathrm{GeV})$ & $m_{H^{+}}(\mathrm{GeV})$ & $m_{12}(\mathrm{GeV})$ & Perturbative till \\
\hline BP1 & 1.78 & 354 & 380 & 341 & 222 & $\sim 10^{7} \mathrm{GeV}$ \\
BP2 & 2.50 & 489 & 506 & 486 & 286 & $\sim 10^{11} \mathrm{GeV}$ \\
BP3 & 7.28 & 320 & 297 & 324 & 117 & $\sim 10^{16} \mathrm{GeV}$ \\
BP4 & 8.28 & 500 & 500 & 500 & 172 & $\sim 10^{19} \mathrm{GeV}$ \\
BP5 & 6.90 & 501 & 499 & 500 & 189 & $\sim 10^{19} \mathrm{GeV}$ \\
BP6 & 10.94 & 1499 & 1500 & 1498 & 451 & $\sim 10^{19} \mathrm{GeV}$ \\
\hline
\end{tabular}

\subsubsection{Higgs signal strengths}

For a 'near-aligned' $2 \mathrm{HDM}$, i.e. that has $(\beta-\alpha) \simeq \frac{\pi}{2}$, signal strengths of $h$ to the fermionic and $V V$ (Here $V=W, Z$ ) final states [49] nearly coincide with the corresponding SM values [50,51]. What can still deviate, is the strength of the $h \gamma \gamma$ interaction. This is attributed to the participation of the charged Higgs in the loop [32,52-55]. The corresponding decay width is given by

$$
\begin{aligned}
\Gamma^{2 H D M}(h \rightarrow \gamma \gamma)= & \frac{\alpha^{2} g^{2}}{2^{10} \pi^{3}} \frac{m_{h}^{3}}{M_{W}^{2}} \mid \sin (\beta-\alpha) F_{W} \\
& +\left(\frac{\cos \alpha}{\sin \beta}\right) \frac{4}{3} F_{t}+\left.\kappa F_{H^{+}}\right|^{2} .
\end{aligned}
$$

Here $g_{2}$ denotes the $S U(2)_{L}$ gauge coupling. Also, $\kappa$ is a dimensionless parameter that quantifies the strength of the coupling of $h$ to a pair of charged Higgses. More clearly,

$g_{h H^{+} H^{-}}=\kappa \frac{g_{2} m_{H^{+}}^{2}}{m_{W}}$.

Here $g_{h H^{+} H^{-}}$denotes the above mentioned trilinear coupling. The functions $F_{W}, F_{t}$ and $F_{i+}$ encapsulate the effects of a W-boson, a t-quark and a charged scalar running in the loop and shall be defined as

$$
\begin{aligned}
F_{W} & =2+3 \tau_{W}+3 \tau_{W}\left(2-\tau_{W}\right) f\left(\tau_{W}\right), \\
F_{t} & =-2 \tau_{t}\left[1+\left(1-\tau_{t}\right) f\left(\tau_{t}\right)\right], \\
F_{H^{+}} & =-\tau_{i+}\left[1-\tau_{i+} f\left(\tau_{i+}\right)\right], \\
f(\tau) & =\left[\sin ^{-1}(\sqrt{1 / \tau})\right]^{2}, \\
\text { with } \tau & =\frac{4 m_{a}^{2}}{m_{h}^{2}}
\end{aligned}
$$

Here $a=t, W$ and $H^{+}$.

We assume $h$ is dominantly produced through gluon fusion. In such a case, the signal strength for the diphoton final state is approximately given by

$\mu_{\gamma \gamma}=\frac{\Gamma^{2 H D M}(h \rightarrow \gamma \gamma)}{\Gamma^{S M}(h \rightarrow \gamma \gamma)}$.
In order to respect the $2 \sigma$ bound on $\mu_{\gamma \gamma}$, from a combined measurement of ATLAS and CMS, we discard model points that violate $\mu_{\gamma \gamma} \in[1.04,1.37]$ [56].

\section{Results and discussions}

Model points that successfully negotiate all the aforesaid constraints are allowed to evolve under RG, till some scale $\Lambda$ (say). $\Lambda$ can be interpreted as the scale up to which no physics over and above the extended Higgs sector is required. If there is an additional, lower vacuum before $\Lambda$, the time scale for tunnelling from the EW vacua to the new one must therefore be larger than the age of the universe. It is intuitively expected that the higher is $\Lambda$, the tighter becomes the parameter space that is allowed at the electroweak scale. This is indeed confirmed by the findings reported in $[7,11]$. Of course, the points leading to a metastable EW vacuum are identified through a detailed scan of the parameter space. However, the fate of a particular model point at high scales is sensitive to the value of the top quark mass taken. With this in view, we propose the benchmarks listed in Table 1.

In each case, we plot the evolution of $\lambda_{2}^{\text {eff }}$ in Fig. 1 . The chosen benchmarks differ in their perturbative behaviour at high scales, although all of them have the common feature that the EW vacuum turns metastable, or even unstable for $M_{t}=175 \mathrm{GeV}$. In other words, the Type-II 2HDM may turn non-perturbative beyond a scale $\Lambda$, even though a vacuum deeper than the EW might be encountered some place intermediate between the electroweak scale and $\Lambda$. Besides, although it is worth identifying those parameter points that keep the EW vacuum metastable all the way till the GUT or Planck scales, we also include for completeness in the benchmarks, two points where a 2HDM loses its perturbativity at a much lower scale. For instance in BP1 (Fig. 1a), at $M_{t}=175 \mathrm{GeV}, \lambda_{2}^{\text {eff }}$ turns negative and, $\frac{\mathrm{d} \lambda_{2}^{\text {eff }}}{\mathrm{d} t}=0$ occurs around $6.2 \times 10^{6} \mathrm{GeV}$ (the scale at which the tunnelling probability gets maximised). Inspection of Fig. 1a thus indicates that this particular benchmark leads to metastability. The same parameter point offers absolute stability for $M_{t}=171 \mathrm{GeV}$ though. BP2 (Fig. 1b) describes a similar 


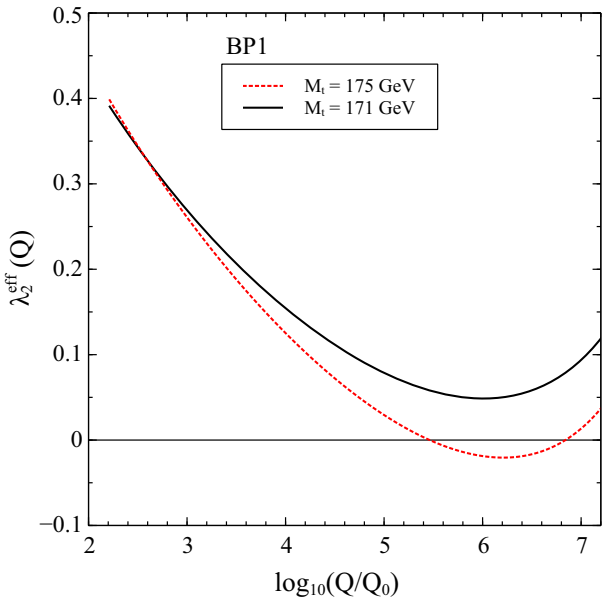

(a)

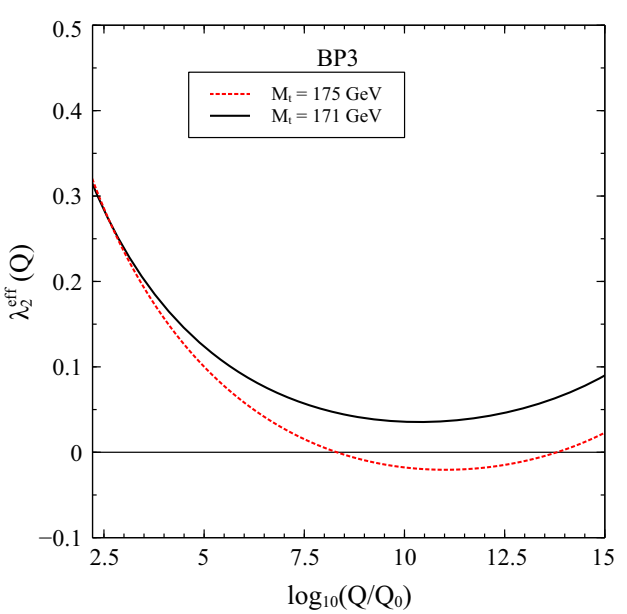

(c)

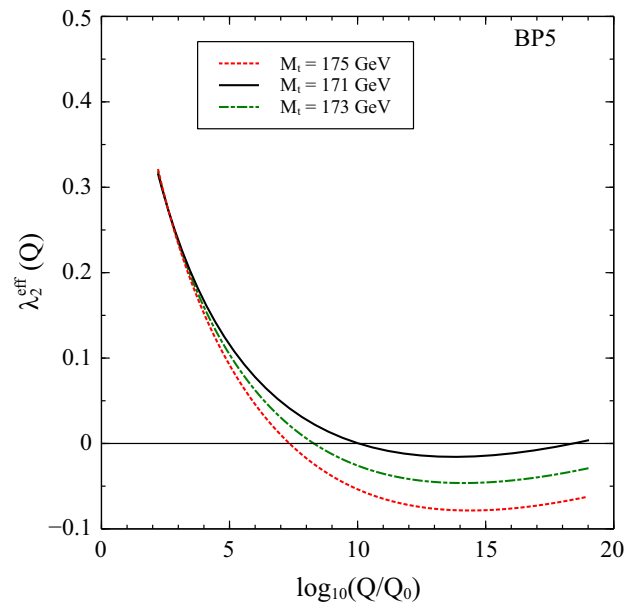

(e)

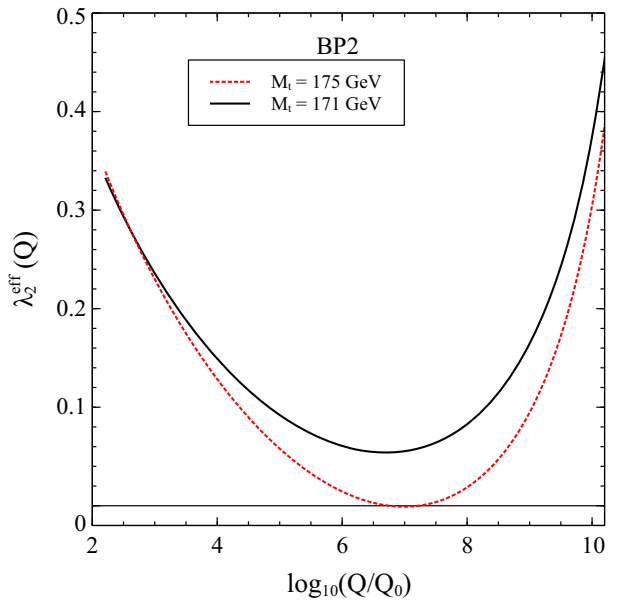

(b)

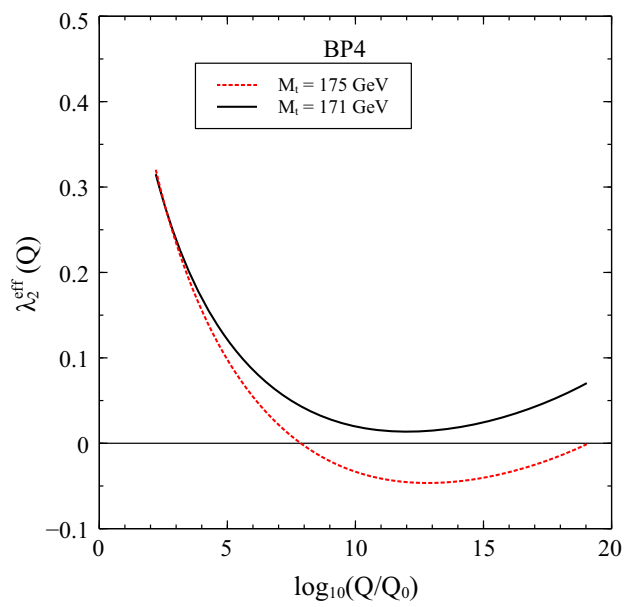

(d)

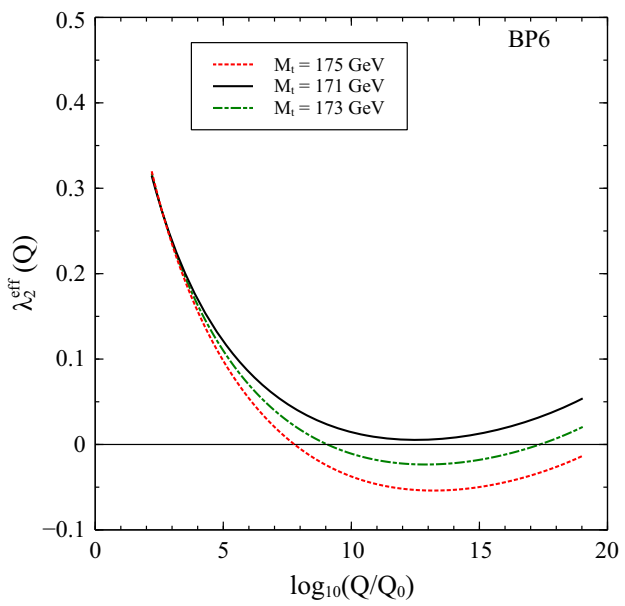

(f)

Fig. 1 RG evolution of $\lambda_{2}^{\text {eff }}$ for the benchmarks listed in Table 1, for more than one value of $M_{t}$. The colour coding is explained in the legends 


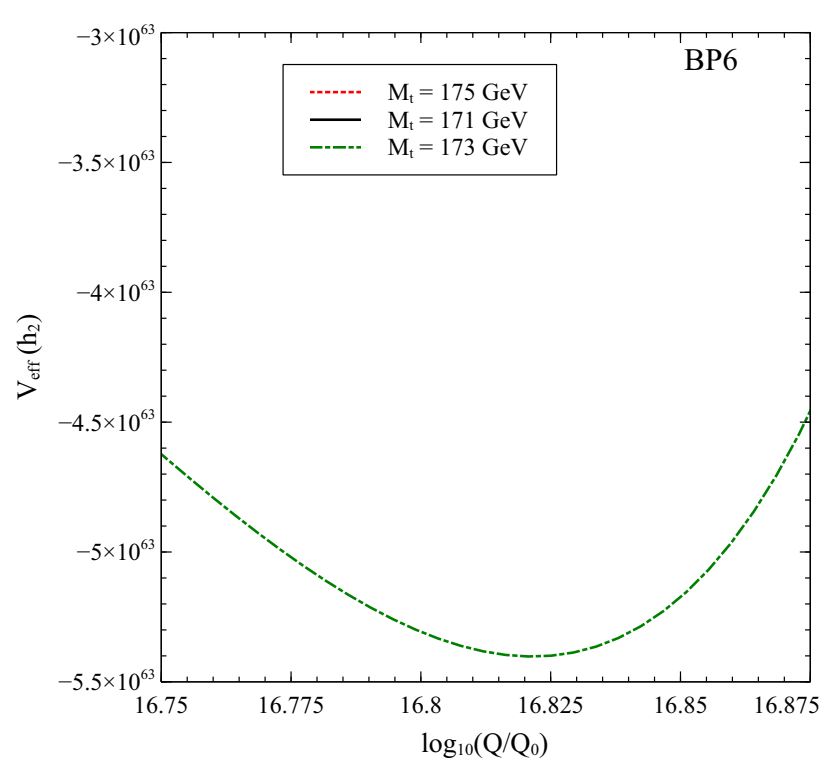

Fig. 2 Behaviour of $V_{\text {eff }}\left(h_{2}\right)$ in BP6 for $M_{t}=173 \mathrm{GeV}$

qualitative feature as seen in BP1, it also remains perturbative till $10^{11} \mathrm{GeV}$.

BP3 (Fig. 1c) is a more conservative benchmark in the sense that it keeps the 2HDM perturbative till the GUT scale and also prevents an unstable EW vacuum even in the worst case scenario with $M_{t}=175 \mathrm{GeV}$. We remind the reader that the strength of the top quark Yukawa coupling depends not only on the pole mass, but also on $\tan \beta$. This becomes crucial in deciding the fate of the EW vacuum at high scales. For instance, BP5 (Fig. 1e) experiences a higher t-quark negative pull compared to BP4 (Fig. 1d) owing to a lower value of $\tan \beta$ in BP5, even though the quartic couplings at the input scale are at the same ball-park for the two cases. BP6 (Fig. 1f) is a fine-tuned parameter point that is perturbative till the Planck scale, and for which the EW vacuum is stable, metastable or unstable for $M_{t}=171 \mathrm{GeV}, 173 \mathrm{GeV}$ and $175 \mathrm{GeV}$, respectively. For the sake of completeness, we display the behaviour $V_{\text {eff }}\left(h_{2}\right)$ for the BP6 benchmark with $M_{t}=173 \mathrm{GeV}$ in Fig. 2.

Model points are randomly sampled in the following specified ranges:

$$
\begin{aligned}
\tan \beta & \in[0.1,20.0], \\
m_{H}, m_{A}, m_{H^{+}}, m_{12} & \in[0,1200 \mathrm{GeV}] .
\end{aligned}
$$

A condition forbidding the loss of perturbativity/unitarity at scale $\Lambda$ is imposed throughout the scan. The following broad features emerge from Figs. 3, 4, and 5. The results are shown using two representative values of $\Lambda$, namely $10^{16} \mathrm{GeV}$ (Fig. 3) and $\Lambda=10^{19} \mathrm{GeV}$ (Fig. 4).

(i) Perturbativity puts stringent constraints on the splitting amongst the masses. In fact, for $\Lambda=10^{19} \mathrm{GeV}$ the masses are near-degenerate (see Fig. 5). This effect can be attributed to the fact that, for a large mass splitting, the $\lambda_{i}$ are already large at the electroweak scale, leading to a blow-up soon after. In fact, a parameter point ensuring perturbative behaviour till the Planck scale does not tolerate a mass splitting higher than $\simeq 10 \mathrm{GeV}$. This constraint in considerably stronger than that from the T-parameter alone $(\simeq 50 \mathrm{GeV})$. In other words, as far as the extended scalar potential is concerned, the Tparameter constraint in thus rendered redundant by the requirement of perturbativity till the GUT or the Planck scale.

(ii) As we mostly confine ourselves to a conservatively perturbative regime $\left(\left|\lambda_{i}\right|<1\right)$, the results obtained using two-loop evolution were found to differ only slightly from the corresponding one-loop ones. Thus, the overall conclusions continue to remain the same.

(iii) A smaller $\tan \beta$ for the same $M_{t}$ implies an enhanced fermionic contribution to the evolution of $\lambda_{2}$, and hence it favours a metastable vacuum over an absolutely stable one. Consequently, $\tan \beta$ is bounded from below in order to prevent tunnelling to the lower vacuum. Moreover, one would apprehend that the bound obtained by demanding absolute stability of the EW vacuum to be stronger than the one obtained when one allows for a metastable scenario. For instance, for $M_{t}=171 \mathrm{GeV}$, the lower bounds read $\simeq 2.1$ and $\simeq 2.5$ for the two cases.

(iv) The lower bound on $\tan \beta$ of course depends on the choice of $M_{t}$. For instance the parameter point parametrised in terms of the masses and $\tan \beta$ indeed shall have different evolution trajectories for two different values of $M_{t}$. This is reflected in the upper and lower plots of Fig. 3, where one witnesses a tighter lower bound, for both the "stable" and the "metastable" models. Of course, in this case too, absolute stability yields a stronger bound than metastability. For this value of $M_{t}$, any model with $\tan \beta<2.6$ yields a tunnelling lifetime lower than the age of the universe.

(v) Although the lower bound on $\tan \beta$ should also depend on the $\Lambda$ chosen, it hardly changes with respect to the $10^{16} \mathrm{GeV}$ value for $\Lambda=10^{19} \mathrm{GeV}$. Only the number of allowed points shrinks to some extent, other essential features are unchanged.

In the plane of $\tan \beta$ versus masses, it is expected that a particular parameter point responsible for a metastable EW vacuum can always be found in the vicinity of a point that leads to absolute stability, $\tan \beta \geq 3.0$. This gets confirmed by an inspection of Fig. 4. This can be understood from the fact that any enhanced fermionic contribution due to a higher $\tan \beta$ can always be cancelled by an appropriately increased bosonic contribution through a slight tweak in the masses. Of course, one also has to keep the couplings perturbative in 


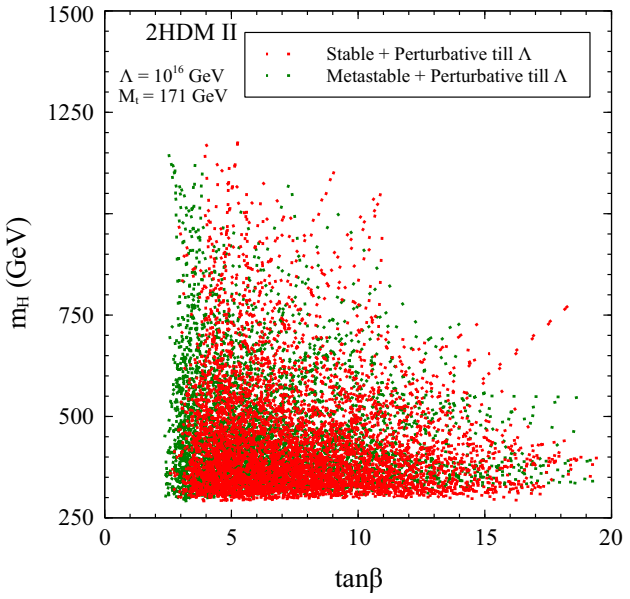

(a)

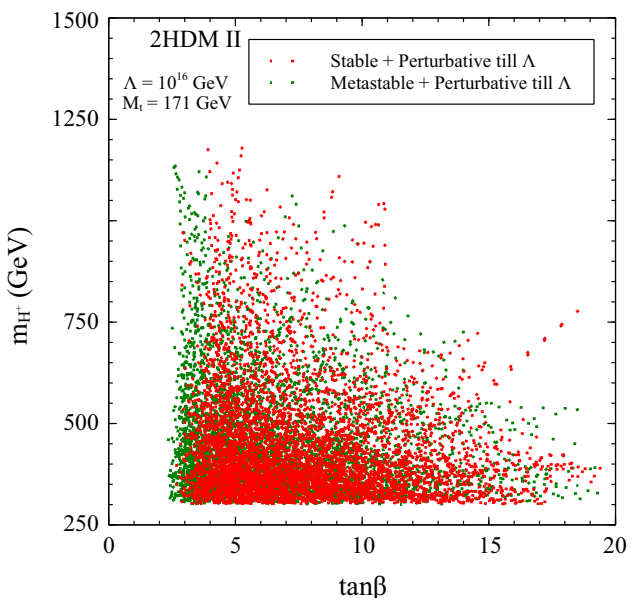

(c)

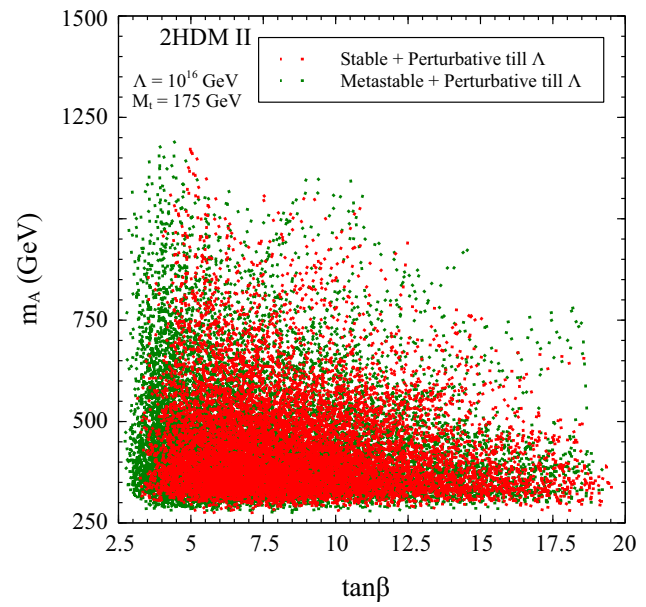

(e)

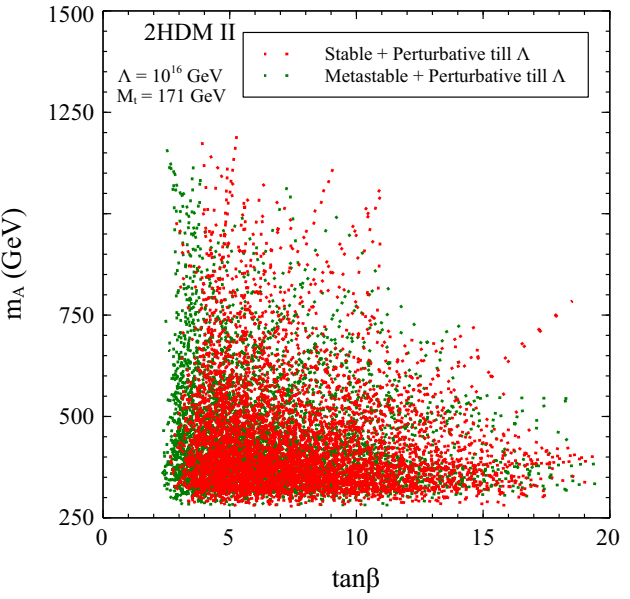

(b)

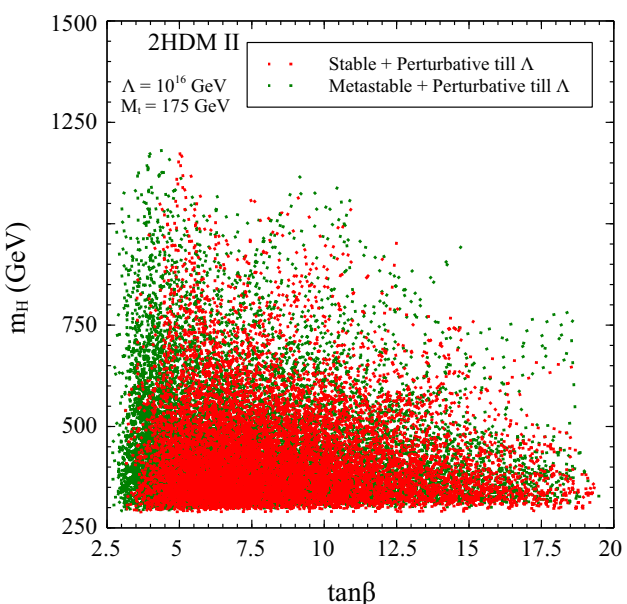

(d)

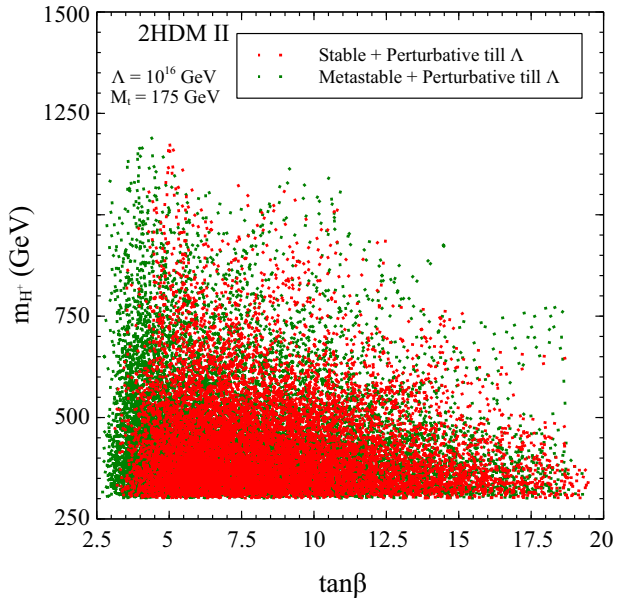

(f)

Fig. 3 Distribution of points in the parameter space perturbative till $10^{16} \mathrm{GeV}$ that lead to an either stable or metastable EW vacuum. The upper (lower) plots correspond to $M_{t}=171(175) \mathrm{GeV}$. The colour coding is explained in the legends. 2HDM II refers to a Type-II 2HDM

doing so. Such a "fine-tuned" existence of a metastable EW vacuum is not a surprise and is always expected in the case of an extended Higgs sector, such as the 2HDM.
We take another approach where different scalar masses are fixed within specific narrow ranges, and we allow $\tan \beta$ to vary. This approach turns useful in demarcating the "sta- 


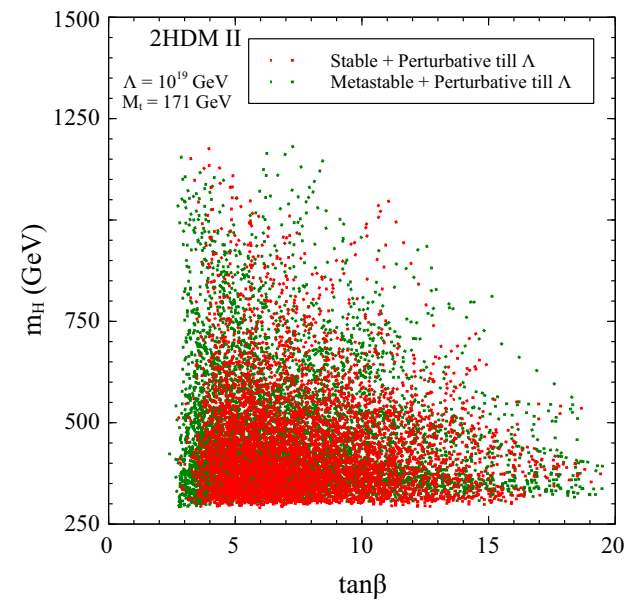

(a)

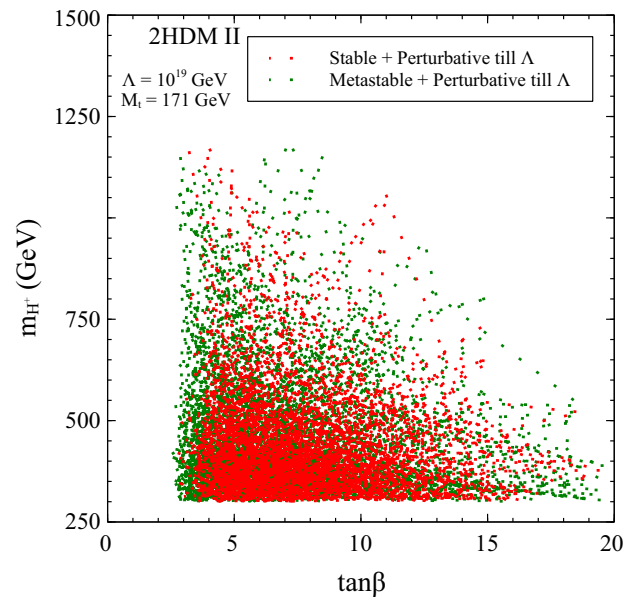

(c)

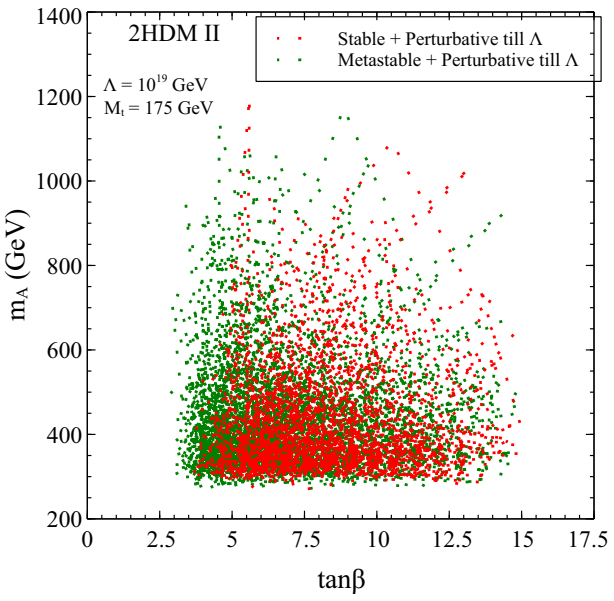

(e)

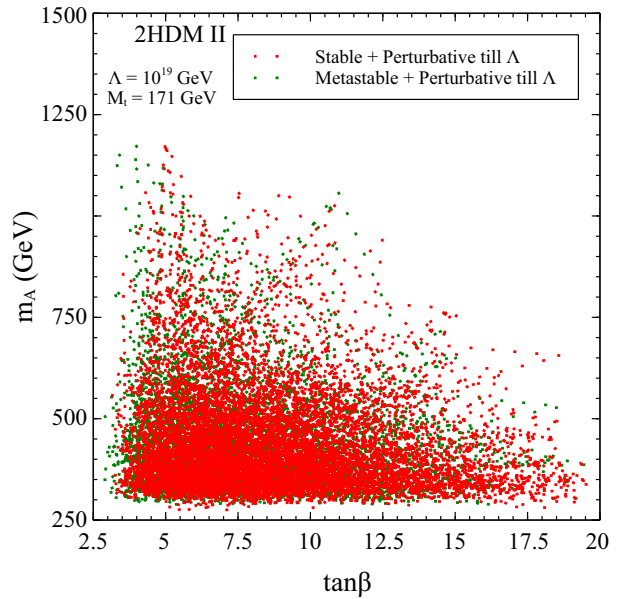

(b)

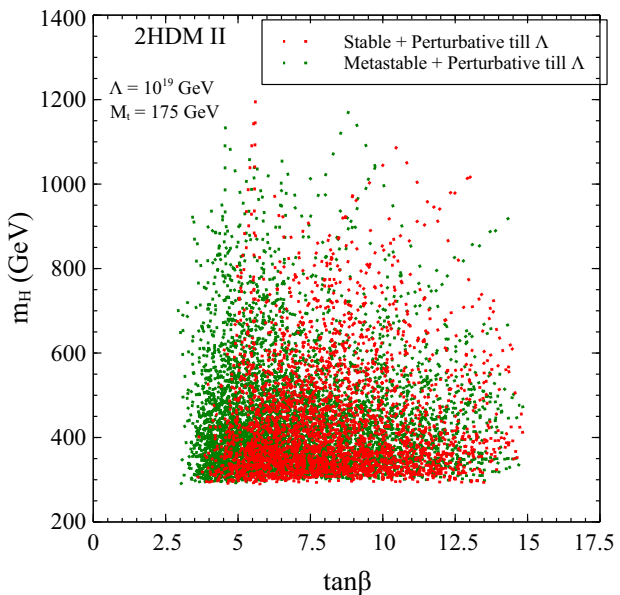

(d)

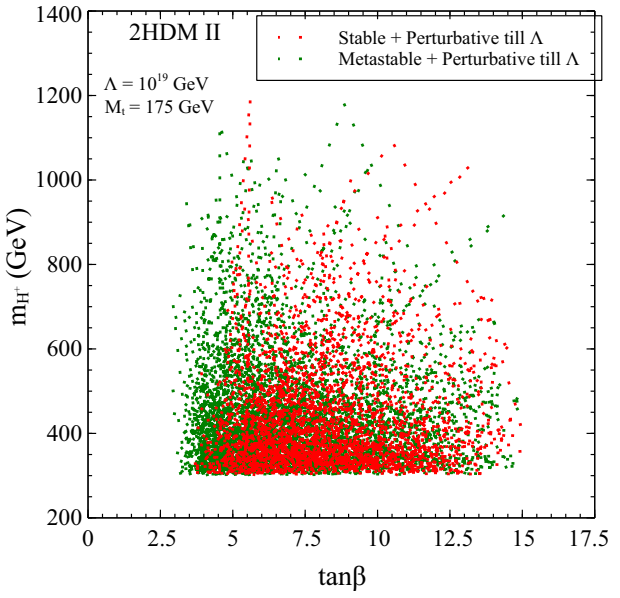

(f)

Fig. 4 Distribution of points in the parameter space perturbative till $10^{19} \mathrm{GeV}$ that lead to an either stable or metastable EW vacuum. The upper (lower) plots correspond to $M_{t}=171(175) \mathrm{GeV}$. The colour coding is explained in the legends. 2HDM II refers to a Type-II 2HDM

ble" region from the "metastable". We thus propose the two central values of 500 and $1000 \mathrm{GeV}$ and allow only a $2 \mathrm{GeV}$ split about that. Figure 6 presents the results for this choice.
For masses around $500 \mathrm{GeV}$ and $M_{t}=171 \mathrm{GeV}$, the metastable points mostly cluster in the low $\tan \beta$ region. They get largely disfavoured at larger $\tan \beta$. Since the bosonic 


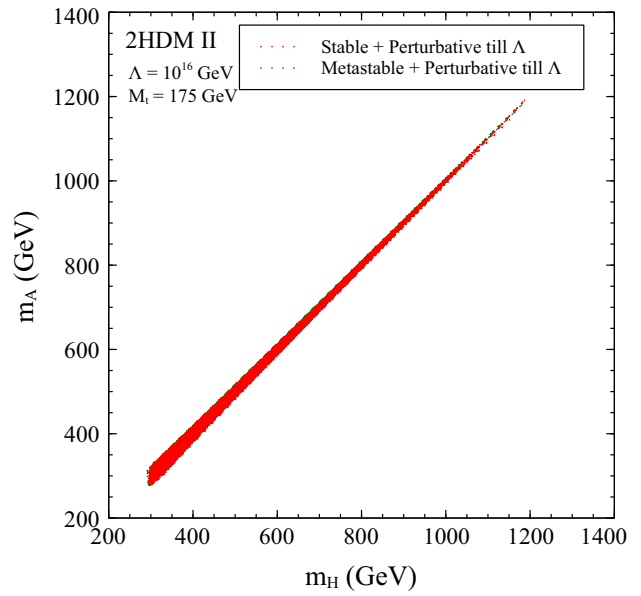

(a)

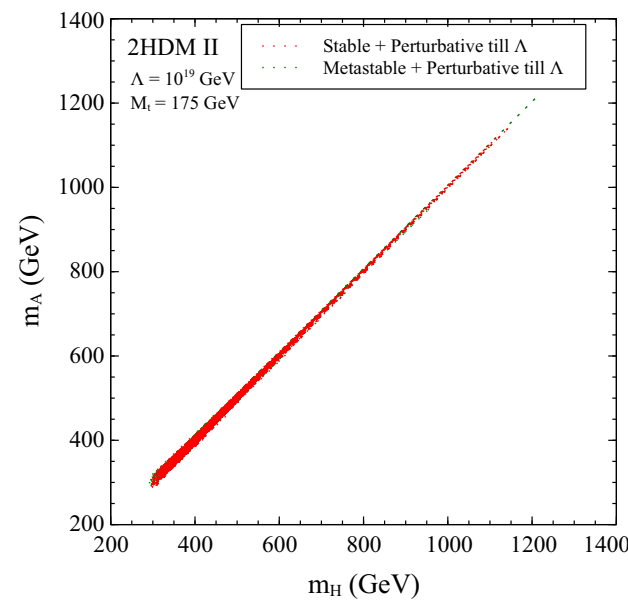

(c)

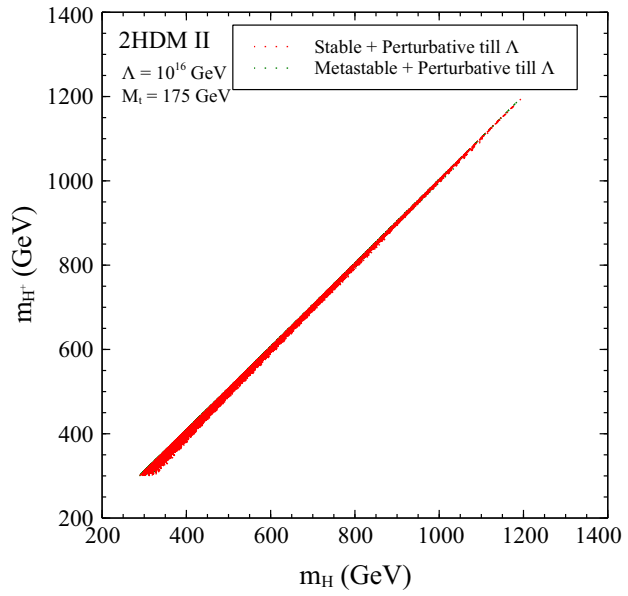

(b)

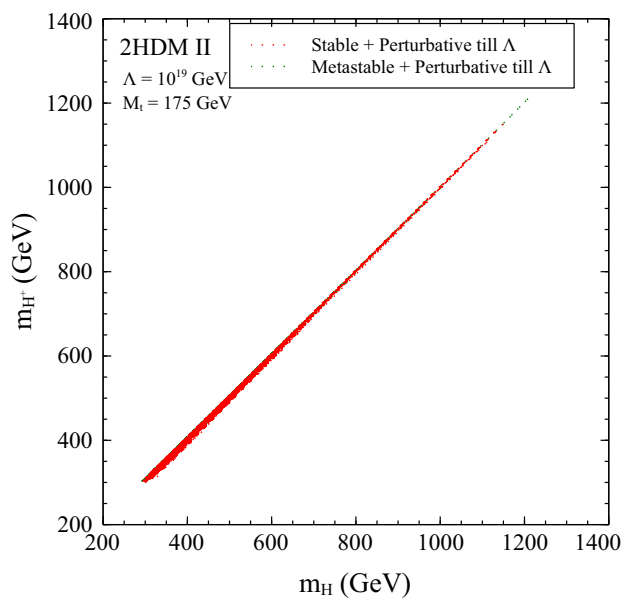

(d)

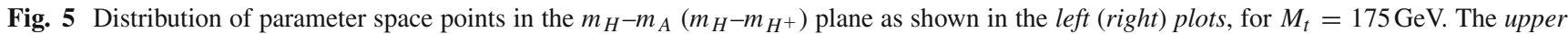
(lower) plots correspond to perturbativity till $10^{16}\left(10^{19}\right) \mathrm{GeV}$

contribution to $\mathrm{RG}$ evolution is now restrained, absolute stability demands $\tan \beta \geq 3.0$. For $M_{t}=175 \mathrm{GeV}$, however, the lower bound on $\tan \beta$ for both stability and metastability increases, and stability is completely ruled out for $\tan \beta \leq$ 5.0 for instance. Thus, for $M_{t}=175 \mathrm{GeV}$, the proportion of metastable model points is higher compared to what is seen for $M_{t}=175 \mathrm{GeV}$. The robustness of this claim is verified by the plots for masses $\simeq 1000 \mathrm{GeV}$, which depict the same qualitative behaviour. Having pointed out the crucial role played by the parameter $\tan \beta$, we close this section here.

\section{Summary and conclusions}

This work highlights the possibility of a metastable EW vacuum in a popular 2HDM framework. We have already noted in Sect. 3 that the LHC data on the $125 \mathrm{GeV}$ Higgs disfavours the occurrence of a panic vacuum at low energy. However, once the parameters occurring in the scalar potential are subject to RG evolution, additional global minima may indeed occur at high scales. This can render the electroweak vacuum unstable or metastable. This is found to happen in the direction of the scalar field $h_{2}$, because $\lambda_{2}$ can be driven to negative values by the top-Yukawa coupling, at high scales. We have reported our findings in the context of the Type-II 2HDM.

We remark that it is the relative strengths of the fermionic and bosonic contributions in the RG improved potential that seals the fate of the EW vacuum where we currently reside. The introduction of additional bosonic degrees of freedom further introduces a tension between vacuum stability on the one hand, and high-scale perturbativity on the other. This tension can be responsible for substantial constraints on the parameter space.

In a 2 HDM, the strength of the fermionic contribution is controlled by not only the top quark pole mass, but also $\tan \beta$. 


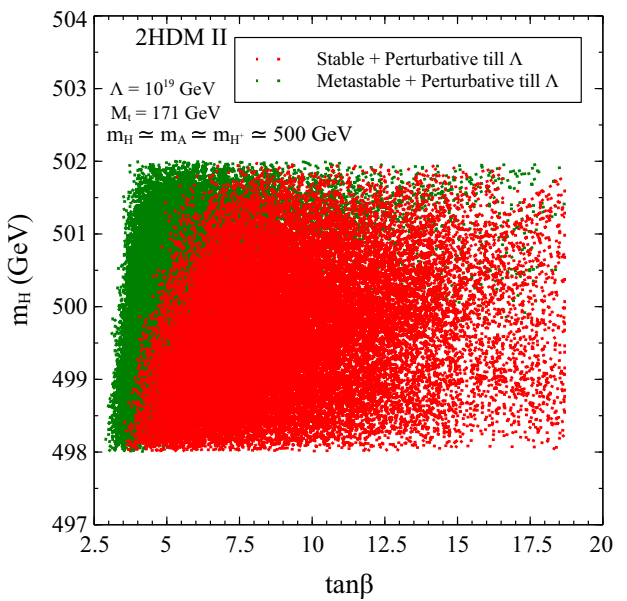

(a)

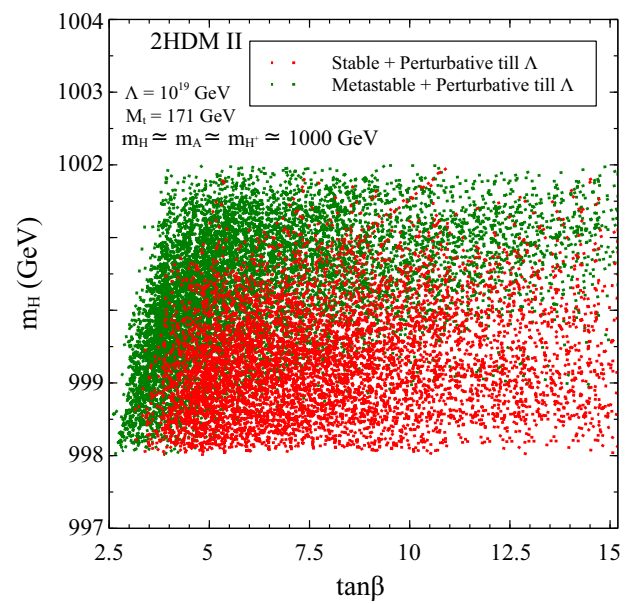

(c)

Fig. 6 Distribution of points in the parameter space in the $m_{H}-\tan \beta$ plane that are perturbative till $10^{19} \mathrm{GeV}$ and lead to an either stable or metastable EW vacuum. The mass splitting amongst the non-standard

Based on the results of this work, one would always expect a metastable model point in the vicinity of a point allowing for absolute stability. However, $\tan \beta$ picks up a lower bound from the requirement of metastability, which is tightened when one demands absolute stability of the EW vacuum. The sensitivity of the results to the top pole mass has also been emphasised.

Side by side with the issue of stability or metastability of the EW vacuum, a crucial role is played by perturbativity and unitarity. A demand of these, for example, all the way to the Planck scale results in the prediction of closely degenerate states $H, A$ and $H^{+}$. We find that this requirement cannot be compromised, even though a larger area of the 2HDM parameter space opens up, on allowing a metastable EW vacuum.

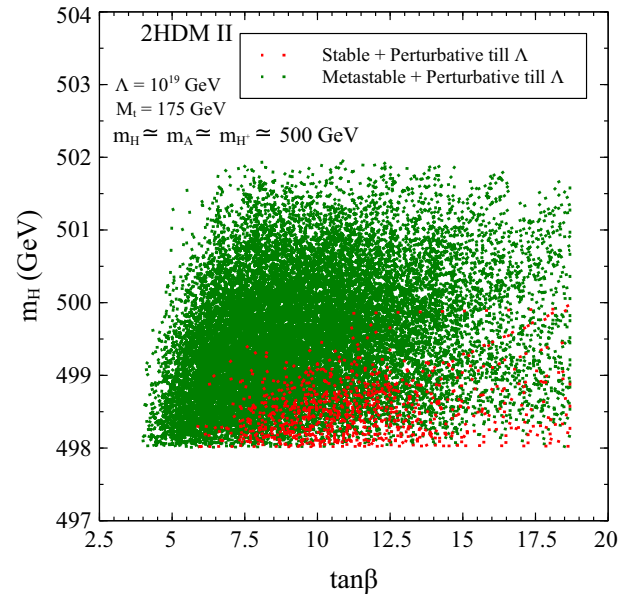

(b)

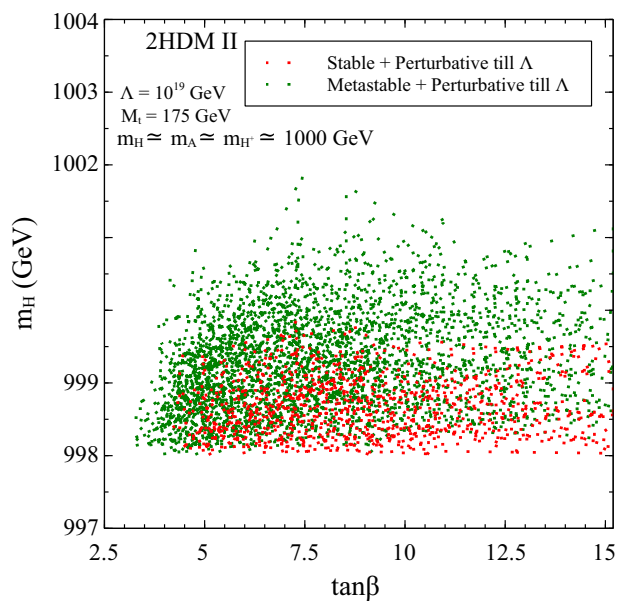

(d)

scalars is forced to stay within $2 \mathrm{GeV}$ during these scans. The upper (lower) plots correspond to $M_{t}=171(175) \mathrm{GeV}$. The colour coding is explained in the legends. 2HDM II refers to a Type-II 2HDM

A pertinent extension would be to include finite temperature corrections to the 2HDM scalar potential and, study its impact on vacuum stability.

Acknowledgements We thank Ashoke Sen for useful discussions. This work was partially supported by funding available from the Department of Atomic Energy, Government of India, for the Regional Centre for Accelerator-based Particle Physics (RECAPP), Harish-Chandra Research Institute.

Open Access This article is distributed under the terms of the Creative Commons Attribution 4.0 International License (http://creativecomm ons.org/licenses/by/4.0/), which permits unrestricted use, distribution, and reproduction in any medium, provided you give appropriate credit to the original author(s) and the source, provide a link to the Creative Commons license, and indicate if changes were made.

Funded by SCOAP ${ }^{3}$. 


\section{References}

1. ATLAS Collaboration, G. Aad et. al., Observation of a new particle in the search for the Standard Model Higgs boson with the ATLAS detector at the LHC. Phys.Lett. B716, 1-29 (2012). arXiv: 1207.7214

2. CMS Collaboration, S. Chatrchyan et al., Observation of a new boson at a mass of $125 \mathrm{GeV}$ with the CMS experiment at the LHC. Phys. Lett. B 716, 30-61 (2012). arXiv:1207.7235

3. G. Degrassi, S. Di Vita, J. Elias-Miro, J.R. Espinosa, G.F. Giudice, G. Isidori, A. Strumia, Higgs mass and vacuum stability in the standard model at NNLO. JHEP 08, 098 (2012). arXiv:1205.6497

4. D. Buttazzo, G. Degrassi, P.P. Giardino, G.F. Giudice, F. Sala, A. Salvio, A. Strumia, Investigating the near-criticality of the Higgs boson. JHEP 12, 089 (2013). arXiv: 1307.3536

5. Particle Data Group Collaboration, K.A. Olive et al, Review of particle physics. Chin. Phys. C 38, 090001 (2014)

6. G. Isidori, G. Ridolfi, A. Strumia, On the metastability of the standard model vacuum. Nuclear Phys. B 609(3), 387-409 (2001)

7. N. Chakrabarty, U.K. Dey, B. Mukhopadhyaya, High-scale validity of a two-Higgs doublet scenario: a study including LHC data. JHEP 12, 166 (2014). arXiv:1407.2145

8. P. Ferreira, H.E. Haber, E. Santos, Preserving the validity of the Two-Higgs doublet model up to the Planck scale. Phys. Rev. D 92, 033003 (2015). arXiv: 1505.0400

9. E.R. Santos, Renormalization group constraints on the two-Higgs doublet model. PhD thesis, UC, Santa Cruz (2015)

10. S. Kanemura, T. Kasai, Y. Okada, Mass bounds of the lightest cpeven higgs boson in the two-higgs-doublet model. Phys. Lett. B 471(23), 182-190 (1999)

11. D. Das, I. Saha, Search for a stable alignment limit in twoHiggs-doublet models. Phys. Rev. D 91(9), 095024 (2015). arXiv: 1503.0213

12. O. Eberhardt, Fitting the Two-Higgs-Doublet model of type II. In: Proceedings, 49th rencontres de Moriond on electroweak interactions and unified theories, pp. 523-526 (2014). arXiv:1405.3181

13. N. Chakrabarty, D.K. Ghosh, B. Mukhopadhyaya, I. Saha, Dark matter, neutrino masses and high scale validity of an inert Higgs doublet model. Phys. Rev. D 92(1), 015002 (2015). arXiv: 1501.0370

14. V. Branchina, E. Messina, Stability, Higgs Boson Mass and new physics. Phys. Rev. Lett. 111, 241801 (2013). arXiv:1307.5193

15. V. Branchina, E. Messina, M. Sher, Lifetime of the electroweak vacuum and sensitivity to planck scale physics. Phys. Rev. D 91, $013003(2015)$

16. N. Khan, S. Rakshit, Constraints on inert dark matter from the metastability of the electroweak vacuum. Phys. Rev. D 92, 055006 (2015). arXiv: 1503.0308

17. N.G. Deshpande, E. Ma, Pattern of symmetry breaking with two Higgs doublets. Phys. Rev. D 18, 2574 (1978)

18. B. Grzadkowski, O.M. Ogreid, P. Osland, Diagnosing CP properties of the 2HDM. JHEP 01, 105 (2014). arXiv:1309.6229

19. J. Shu, Y. Zhang, Impact of a CP violating Higgs sector: from LHC to baryogenesis. Phys. Rev. Lett. 111(9), 091801 (2013). arXiv: 1304.0773

20. C.S. Kim, Y.W. Yoon, X.-B. Yuan, Exploring top quark fenc within $2 \mathrm{hdm}$ type iii in association with flavor physics. J. High Energy Phys. 2015(12), 1-30 (2015)

21. A. Crivellin, A. Kokulu, C. Greub, Flavor-phenomenology of twoHiggs-doublet models with generic Yukawa structure. Phys. Rev. D 87(9), 094031 (2013). arXiv: 1303.5877

22. I. Baum, G. Eilam, S. Bar-Shalom, Scalar flavor changing neutral currents and rare top quark decays in a two $\mathrm{H}$ iggs doublet model 'for the top quark'. Phys. Rev. D 77, 113008 (2008). arXiv:0802.2622
23. S.L. Glashow, S. Weinberg, Natural conservation laws for neutral currents. Phys. Rev. D 15, 1958-1965 (1977)

24. E.A. Paschos, Diagonal neutral currents. Phys. Rev. D 15, 19661972 (1977)

25. G. Cvetič, S.S. Hwang, C.S. Kim, Higgs-mediated flavor-changing neutral currents in the general framework with two higgs doublets: An rge analysis. Phys. Rev. D 58, 116003 (1998)

26. A. Barroso, P.M. Ferreira, I.P. Ivanov, R. Santos, Metastability bounds on the two Higgs doublet model. JHEP 06, 045 (2013). arXiv: 1303.5098

27. I. Chakraborty, A. Kundu, Scalar potential of two-Higgs doublet models. Phys. Rev. D 92(9), 095023 (2015). arXiv: 1508.0070

28. S. Coleman, E. Weinberg, Radiative corrections as the origin of spontaneous symmetry breaking. Phys. Rev. D 7, 1888-1910 (1973)

29. J.S. Lee, A. Pilaftsis, Radiative corrections to scalar masses and mixing in a scale invariant two Higgs doublet model. Phys. Rev. D 86, 035004 (2012). arXiv:1201.4891

30. S. Coleman, Fate of the false vacuum: semiclassical theory. Phys. Rev. D 15, 2929-2936 (1977)

31. C.G. Callan, S. Coleman, Fate of the false vacuum. ii. first quantum corrections. Phys. Rev. D 16, 1762-1768 (1977)

32. G. Branco, P. Ferreira, L. Lavoura, M. Rebelo, M. Sher et al., Theory and phenomenology of two-Higgs-doublet models. Phys. Rep. 516, 1-102 (2012). arXiv:1106.0034

33. J. Bernon, J.F. Gunion, H.E. Haber, Y. Jiang, S. Kraml, Scrutinizing the alignment limit in two-higgs-doublet models: $m_{h}=125 \mathrm{GeV}$. Phys. Rev. D 92, 075004 (2015)

34. J. Bernon, J.F. Gunion, H.E. Haber, Y. Jiang, S. Kraml, Scrutinizing the alignment limit in two-Higgs-doublet models: $\mathrm{m}_{h}=125 \mathrm{GeV}$. Phys. Rev. D 92(7), 075004 (2015). arXiv: 1507.0093

35. A.G. Akeroyd, A. Arhrib, E.-M. Naimi, Note on tree level unitarity in the general two Higgs doublet model. Phys. Lett. B 490, 119-124 (2000). arXiv:hep-ph/0006035

36. J. Horejsi, M. Kladiva, Tree-unitarity bounds for THDM Higgs masses revisited. Eur. Phys. J. C 46, 81-91 (2006). arXiv:hep-ph/0510154

37. S. Kanemura, K. Yagyu, Unitarity bound in the most general two Higgs doublet model. Phys. Lett. B 751, 289-296 (2015). arXiv: 1509.0606

38. I.F. Ginzburg, I.P. Ivanov, Tree-level unitarity constraints in the most general 2HDM. Phys. Rev. D 72, 115010 (2005). arXiv:hep-ph/0508020

39. S. Kanemura, T. Kubota, E. Takasugi, Lee-Quigg-Thacker bounds for Higgs boson masses in a two doublet model. Phys. Lett. B 313, 155-160 (1993). arXiv:hep-ph/9303263

40. P. Ferreira, R. Santos, A. Barroso, Stability of the tree-level vacuum in two Higgs doublet models against charge or $\mathrm{CP}$ spontaneous violation. Phys. Lett. B 603, 219-229 (2004). arXiv:hep-ph/0406231

41. N.G. Deshpande, E. Ma, Pattern of symmetry breaking with two higgs doublets. Phys. Rev. D 18, 2574-2576 (1978)

42. S. Nie, M. Sher, Vacuum stability bounds in the two-higgs doublet model. Phys. Lett. B 449(12), 89-92 (1999)

43. M.E. Peskin, T. Takeuchi, Estimation of oblique electroweak corrections. Phys. Rev. D 46, 381-409 (1992)

44. G. Funk, D. O'Neil, R.M. Winters, What the oblique parameters S, $\mathrm{T}$, and $\mathrm{U}$ and their extensions reveal about the 2HDM: a numerical analysis. Int. J. Mod. Phys. A 27, 1250021 (2012). arXiv: 1110.3812

45. D. Toussaint, Renormalization effects from superheavy higgs particles. Phys. Rev. D 18, 1626-1631 (1978)

46. H.E. Haber, D. O'Neil, Basis-independent methods for the twoHiggs-doublet model III: the CP-conserving limit, custodial symmetry, and the oblique parameters S, T, U. Phys. Rev. D 83, 055017 (2011). arXiv:1011.6188 
47. Gfitter Group Collaboration, M. Baak et al, The global electroweak fit at NNLO and prospects for the LHC and ILC. Eur. Phys. J. C 74, 3046 (2014). arXiv: 1407.3792

48. F. Mahmoudi, O. Stal, Flavor constraints on the two-Higgs-doublet model with general Yukawa couplings. Phys. Rev. D 81, 035016 (2010). arXiv:0907.1791

49. K. Cheung, J.S. Lee, P.-Y. Tseng, Higgcision in the two-Higgs doublet models. JHEP 01, 085 (2014). arXiv:1310.3937

50. O. Eberhardt, U. Nierste, M. Wiebusch, Status of the two-Higgsdoublet model of type II. JHEP 07, 118 (2013). arXiv:1305.1649

51. H.S. Cheon, S.K. Kang, Constraining parameter space in type-II two-Higgs doublet model in light of a $126 \mathrm{GeV}$ Higgs boson. JHEP 09, 085 (2013). arXiv: 1207.1083

52. M.A. Shifman, A.I. Vainshtein, M.B. Voloshin, V.I. Zakharov, Low-energy theorems for Higgs Boson couplings to photons. Sov. J. Nucl. Phys. 30, 711-716 (1979)
53. M.A. Shifman, A.I. Vainshtein, M.B. Voloshin, V.I. Zakharov, Yad. Fiz. 30, 1368 (1979)

54. H. Baer, M. Bisset, C. Kao, X. Tata, Observability of $\gamma \gamma$ decays of higgs bosons from supersymmetry at hadron supercolliders. Phys. Rev. D 46, 1067-1075 (1992)

55. P. Kalyniak, R. Bates, J.N. Ng, Two-photon decays of scalar and pseudoscalar bosons in supersymmetry. Phys. Rev. D 33, 755-762 (1986)

56. ATLAS Collaboration, G. Aad et. al., Measurement of Higgs boson production in the diphoton decay channel in pp collisions at centerof-mass energies of 7 and $8 \mathrm{TeV}$ with the ATLAS detector. Phys. Rev. D90(11), 112015 (2014). arXiv:1408.7084 